Result. All reflections moved away from a technical understanding of schizophrenia, towards person-centred interpretations, with dominant themes of 'stigma', 'disempowerment', 'understanding people as individuals', 'subjective experience of mental illness', 'inclusion' and 'healing power of nature'.

The three prize winners (authors GY, AK and KT) used different mediums: GY painted an osprey over a chaotic collage of disordered and stigmatizing words (the osprey representing empowerment and the "reservoir for wellbeing in nature"); AK's sonnet began as an ode to the chaos of Justin's experience, but the concluding lines reframed this struggle, conveying feelings of hope and beauty; and KT's self-portrait, produced with a slow shutter-speed photograph, powerfully conveyed a sense of disorientation and disturbance. She reflected on how the stigma of mental illness affects self-perception. The talk was wellattended, and reflections were of high quality. A limitation of this pilot was that only a small proportion of students completed the reflective assignment.

Conclusion. Innovative teaching strategies are needed to address negative attitudes towards mental illness and psychiatry, which are prevalent amongst the medical profession. This pilot provides a model for combining carer-led, reflective, and creative elements in undergraduate psychiatry teaching, with the aim of challenging stigma. This model will be evaluated in a further study involving fifth year medical students, which will use a validated scale to measure change in students' attitudes towards mental illness and psychiatry.

\section{An audit on consent to treatment within forensic inpatient units at the Newsam Centre}

Elisabeth Bond ${ }^{*}$, Stephanie Vel En Tial and Clare Stephenson

Leeds and York Partnership NHS Trust

${ }^{*}$ Corresponding author.

doi: 10.1192/bjo.2021.83

Aims. We aimed to investigate the adherence to the Mental Health Act Code of Practice and the adequate documentation of consent to treatment across three forensic low secure inpatient units.

Method. Our sample included all inpatients detained on three forensic wards at The Newsam Centre. This included a total of 31 patients with an age range of 25 to 59 years. The Mental Health Act Code of Practice was used as criteria for audit standards. Data were collected using Microsoft Excel and analysed using descriptive methods.

Result. We found that 28 patients out of 31 had been admitted for over three months and of these patients 12 were subject to a T2 and 16 subject to a T3. A total of 24 patients had their CTT medication list documented on the online drug chart; with a remaining seven patients who did not. As per guidelines, 27 patients had the appropriate medications prescribed as per their CTT, however one patient did not. The audit revealed a total of two patients currently on a Section 62. Of the qualifying T3 forms, four patients had this reviewed every two years whilst there was one patient who had not. Conclusion. We found that the adherence to Mental Health Act Code of Practice was overall positive with the majority of service users being reviewed appropriately and documented as per guidance. However, areas identified for improvement included the recording of CTT on online drug charts as well as reviewing T3 every two years. This audit highlights the need for easy access to guidance, appropriate documentation as well as frequent checking of adherence. A leaflet has been created outlining the guidelines and will be distributed to all staff working within the forensic settings and placed in easily accessible locations. As further recommendations from this audit we advise all wards to plan weekly checks during team meetings to ensure information is up to date and that all staff are aware of any discrepencies. A re-audit is planned in the coming months to re-assess adherance after implementation of the interventions.

\section{Comorbidity of self-harm and disordered eating in} young people: evidence from a UK population-based cohort

Helen Bould ${ }^{1 \star}$, Naomi Warne ${ }^{2}$, Jon Heron², Becky Mars², Paul Moran ${ }^{2}$, Anne Stewart ${ }^{3}$, Marcus Munafo ${ }^{4}$, Lucy Biddle ${ }^{2}$, Andy Skinner ${ }^{5}$ and David Gunnell ${ }^{2}$

${ }^{1}$ Centre for Academic Mental Health, Population Health Sciences, Bristol Medical School, University of Bristol, Gloucestershire Health and Care NHS Foundation Trust, MRC Integrative Epidemiology Unit, University of Bristol Medical School; ${ }^{2}$ Centre for Academic Mental Health, Population Health Sciences, Bristol Medical School, University of Bristol; ${ }^{3}$ Department of Psychiatry, University of Oxford; ${ }^{4}$ School of Psychological Science, University of Bristol, MRC Integrative Epidemiology Unit, University of Bristol Medical School, NIHR Biomedical Research Centre, University Hospitals Bristol NHS Foundation Trust and ${ }^{5}$ School of Psychological Science, University of Bristol, MRC Integrative Epidemiology Unit, University of Bristol Medical School

${ }^{*}$ Corresponding author.

doi: 10.1192/bjo.2021.84

Aims. Self-harm and eating disorders are often comorbid in clinical samples but their co-occurrence in the general population is unclear. Given that only a small proportion of individuals who self-harm or have disordered eating present to clinical services, and that both self-harm and eating disorders are associated with substantial morbidity and mortality, we aimed to study these behaviours at a population level.

Method. We assessed the co-occurrence of self-harm and disordered eating behaviours in 3384 females and 2326 males from a UK population-based cohort: the Avon Longitudinal Study of Parents and Children (ALSPAC). Participants reported on their self-harm and disordered eating behaviours (fasting, purging, binge-eating and excessive exercise) in the last year via questionnaire at 16 and 24 years. At each age we assessed how many individuals who self-harm also reported disordered eating, and how many individuals with disordered eating also reported self-harm. Result. We found high comorbidity of self-harm and disordered eating. Almost two-thirds of 16-year-old females, and two-in-five 24-year-old males who self-harmed also reported some form of disordered eating. Young people with disordered eating reported higher levels of self-harm at both ages compared to those without disordered eating.

Conclusion. As self-harm and disordered eating commonly co-occur in young people in the general population, it is important to screen for both sets of difficulties to provide appropriate treatment.

Exploring the relationship between anxiety, depression and wellbeing in doctors: a national cross-sectional survey and interviews
Emma Boxley ${ }^{1 \star}$, Gemma Simons ${ }^{2}$ and John Jenkins ${ }^{1}$
${ }^{1}$ University of Southampton and ${ }^{2}$ Centre for Workforce Wellbeing,
University of southampton
${ }^{*}$ Corresponding author.

doi: 10.1192/bjo.2021.85 